\title{
Infliximab and serious hematologic events
}

B See related article page 1063

Reason for posting: Remicade (infliximab), a tumour necrosis factor- $\alpha(\mathrm{TNF}-\alpha)$ antagonist, is used in the treatment of rheumatoid arthritis and Crohn's disease. However, the US Food and Drug Administration (FDA) has recently advised of serious hematologic events, including leukopenia, neutropenia, thrombocytopenia and pancytopenia, in some patients taking the drug (www.fda .gov/medwatch/SAFETY/2004 /safety04.htm\#remicade).

The drug: TNF- $\alpha$ is a potent proinflammatory cytokine produced by macrophages in response to infectious or inflammatory stimuli. Its activity has been implicated in many inflammatory disorders, including rheumatoid arthritis, Crohn's disease and psoriasis. ${ }^{1}$ Infliximab is a chimeric monoclonal antibody that targets TNF- $\alpha$, which leads to a disruption of the inf flammatory cascade. Common adverse reactions include dyspnea, urticaria and headache. It is now well recognized that infections, including tuberculosis, invasive fungi and other opportunistic infections, may occur in patients taking the drug. ${ }^{1,2}$ Other possible adverse reactions include exacerbations of multiple sclerosis and demyelination syndromes, congestive heart failure and lupus. ${ }^{2,3}$

The FDA advisory contains no clinical details on the patients affected by blood dyscrasias. The incidence of this adverse effect is unknown, but in some published case reports it seems to occur within a few weeks after treatment is initiated. ${ }^{4,5}$ Blood dyscrasias associated with another TNF- $\alpha$ inhibitor, etanercept, have been fatal: in one case series, in which 3 patients had aplastic anemia and 7 had pancytopenia, 5 of these 10 patients died. ${ }^{3}$ The mechanism by which this effect is occurring is unknown, but the TNF- $\alpha$ blockade may lead to inhibition of proinflammatory cytokines (including interleukin-1, interleukin-6, interleukin-8 and granulocyte colony-stimulating factor) involved in bone marrow stem cell differentiation. ${ }^{5}$ The reasons why only a limited number of patients are affected are still unclear.

What to do: It may be wise to check the patient's baseline complete blood count before and shortly after infliximab therapy is started. It is important to warn patients of all serious adverse effects and to check their complete blood count as required (e.g., in the event of fever, anemic symptoms, bruising and easy bleeding). It is unknown whether intermittent monitoring of complete blood counts when the patient is taking infliximab can prevent serious outcomes.

\section{Cynthia Phelan \\ Resident \\ Department of Family Medicine \\ University of Ottawa \\ Eric Wooltorton \\ CMAF}

\section{References}

1. Long R, Gardam M. Tumour necrosis factor-alpha inhibitors and the reactivation of latent tuberculosis infection. CMA7 2003;168(9):1153-6.

2. Lovinger SP. Use of biologics for rheumatiod arthritis tempered by concerns over safety, cost. $7 A M A$ 2003;289(24):3229-30.

3. Day R. Adverse reactions to TNFalpha inhibitors in rheumatoid arthritis. Lancet 2002;359:540-1.

4. Menon Y, Curcurull E, Espunoza LR. Pancytopenia in a patient with scleroderma treated with infliximab. Rheumatology 2003;42:1273-4.

5. Vidal F, Fontova R, Richart C. Severe neutropenia and thrombocytopenia associated with infliximab. Ann Intern Med 2003;139(3):E238-9.

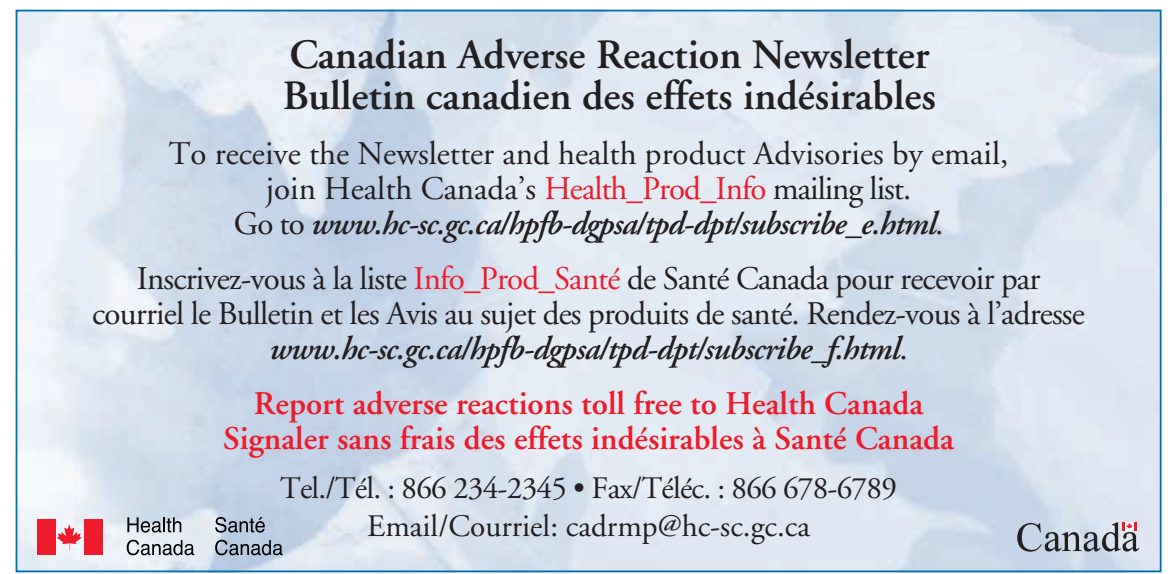

